\title{
Inhibition of centrosomal protein 164 sensitizes rhabdomyosarcoma cells to radiotherapy
}

\author{
JIANZHOU LIU ${ }^{1}$, ZHIJU WANG $^{2}$, XIAOFENG LI $^{1}$, XU ZHANG $^{1}$ and CHAOJI ZHANG ${ }^{1}$ \\ ${ }^{1}$ Department of Cardiac Surgery, Peking Union Medical College Hospital, Chinese Academy of Medical Sciences, \\ Beijing 100730; ${ }^{2}$ Department of Physiology, School of Basic Medicine Science, \\ Zhengzhou University, Zhengzhou, Henan 450001, P.R. China
}

Received December 24, 2015; Accepted January 6, 2017

DOI: $10.3892 /$ etm.2017.4281

\begin{abstract}
Rhabdomyosarcoma is the second most common malignant tumor of the heart in infants and children and cannot often be resected completely. Chemotherapy and radiotherapy have a critical role in relieving symptoms and prolonging survival; therefore, enhancing the sensitivity of rhabdomyosarcoma to radiotherapy is an important area of investigation in order to improve the prognosis of patients. It has been reported that centrosomal protein 164 (CEP164) has a key role in the DNA damage-activated signaling cascade. CEP164 is often overexpressed in tumors and is associated with poor prognosis in various types of cancer. In the present study, the influence of CEP164 on the radiosensitivity of rhabdomyosarcoma cells was investigated. Results demonstrated that CEP164 is involved in the radiation-induced cellular response. CEP164 is increased upon radiation and influences the cell cycle, cell viability and cell apoptosis. CEP164 depletion enhanced cellular sensitivity to radiation, promoted cell apoptosis, decreased cell viability and induced gap $2 /$ mitosis arrest of the cell cycle. The present study identified the function of CEP164 in radiation resistance in rhabdomyosarcoma, providing a potential therapeutic target for rhabdomyosarcoma treatment by disrupting CEP164.
\end{abstract}

\section{Introduction}

The heart is an uncommon organ for primary tumor development. Primary rhabdomyosarcoma of the heart is a rare tumor; however, it is the second most common cardiac sarcoma (1). Rhabdomyosarcoma arises in the right atrium and is often complicated with heart failure, which is serious and difficult to control (2). Early recognition and therapeutic intervention is

Correspondence to: Professor Chaoji Zhang, Department of Cardiac Surgery, Peking Union Medical College Hospital, Chinese Academy of Medical Sciences, 1 Shuaifuyuan, Dongdan, Beijing 100730, P.R. China

E-mail: cjzhang_pumc@163.com

Key words: rhabdomyosarcoma, A-204, A-673, centrosomal protein 164 , radiation extremely important in the prevention of heart failure. Surgery is the mainstay of treatment for non-metastatic disease. If surgical intervention fails to completely resect the tumor $(3,4)$, radiotherapy is essential and effective; however, the therapeutic success of chemotherapy and radiotherapy is poor for metastatic disease $(5,6)$. Therefore, the improvement of radiotherapy success for rhabdomyosarcoma has become a major focus of research.

Proteins of the centrosomal protein (CEP) family are critical components of centrosomes and have vital roles in the control of cell cycle progression. This protein family consists of 31 proteins, one of which is CEP164. CEP164 encodes a $180 \mathrm{kDa}$ protein. CEP164 may be phosphorylated by ataxia telangiectasia mutated (ATM) kinase and Rad3-related protein (ATR) kinase in the DNA damage response (DDR) pathway and has a key role in the gap 2/mitosis (G2/M) checkpoint (7,8). Research has demonstrated that mutations in CEP164 are one cause of nephronophthisis-related ciliopathies (NPHP-RC) (9). In the DDR, CEP164 is rapidly localized to nuclear foci. Research has demonstrated that knockdown of CEP164 induces cell sensitivity to DNA-damaging agents and CEP164 knockdown in zebrafish results in deregulated DDR and an NPHP-RC phenotype (4). Additionally, CEP164 modulates mediator of DNA damage checkpoint protein 1 (MDC1) and checkpoint kinase 1 (CHEK1) to maintain genomic stability (10).

The importance of CEP164 in the DDR makes it a notable target for enhancing the radiosensitivity of cells; however, the effect of combining CEP164 inhibition with irradiation (IR) in rhabdomyosarcoma cells is unknown. In the present study, the role of CEP164 in the cell cycle, and the cell viability of the rhabdomyosarcoma A-204 and A-673 cells lines following treatment with IR were investigated. It was demonstrated that CEP164 expression levels are increased following treatment with IR and depletion of CEP164 enhances cellular sensitivity to radiation, resulting in decreased cell viability, promotion of apoptosis and G2/M cell cycle arrest. In conclusion, the present study demonstrated that CEP164 may be a candidate target gene for rhabdomyosarcoma radiotherapy.

\section{Materials and methods}

Cell culture, small interfering (si)RNA transfection and radiation exposure. Human rhabdomyosarcoma A-204 and A-673 cells (American Type Culture Collection, Manassas, VA, USA) 
were cultured in McCoy's 5A (Modified) Medium supplemented with $10 \%$ fetal bovine serum (FBS; Invitrogen; Thermo Fisher Scientific, Inc., Waltham, MA, USA), incubated at $37^{\circ} \mathrm{C}$ in a $95 \%$ humidified incubator $\left(5 \% \mathrm{CO}_{2}\right)$ and irradiated with doses of 2, 4 or 6 Gy. Cells were treated with Cobalt-60 $\gamma$-rays at $2 \mathrm{~Gy} / \mathrm{min}$ in the Beijing Radiation Center (Beijing Academy of Science and Technology, Beijing, China). Cells were cultured to $80 \%$ confluence and were subsequently transfected with siRNA against the negative control or against CEP164 (Ambion; Thermo Fisher Scientific, Inc.). All transfection was performed using Lipofectamine ${ }^{\circledR}$ RNAiMAX reagent (Thermo Fisher Scientific, Inc.). Cells were exposed to IR $24 \mathrm{~h}$ after target gene knockdown.

Western blot analysis. A-204 cells were lysed in RIPA lysis and extraction buffer containing protease and phosphatase inhibitor (all Pierce; Thermo Fisher Scientific, Inc.) for $20 \mathrm{~min}$ at $4^{\circ} \mathrm{C}$. Protein concentration was measured using a BCA Protein Assay kit (Thermo Fisher Scientific, Inc.), according to the manufacturer's protocol. A total of $30 \mu \mathrm{g}$ of protein was separated by $10 \%$ Bis-Tris gels (Thermo Fisher Scientific, Inc.) and transferred onto nitrocellulose membranes for western blot analysis. Following washing three times with Tris-buffered saline-Tween 20, the membranes were blocked with 5\% nonfat milk for $60 \mathrm{~min}$ and were subsequently incubated with primary anti-CEP164 (1:1,000; GTX85298; GeneTex, Inc., Irvine, CA, USA) or anti- $\beta$-actin antibodies (1:1,000; ab8227; Abcam, Cambridge, UK) overnight at $4^{\circ} \mathrm{C}$. Following two washes with washing buffer $(0.5 \%$ Tween in PBS), the membranes were incubated with horseradish peroxidase-conjugated secondary anti-rabbit antibody $(1: 3,000 ;$ ab6721; Abcam) for $1 \mathrm{~h}$ at room temperature. Following this, protein bands were detected using chemiluminescence liquid (Pierce; Thermo Fisher Scientific, Inc.), according to the manufacturer's instructions, and analyzed using ImageJ software (ImageJ 2x version 2.1.4.6, Bio-Rad Laboratories, Inc., Hercules, CA, USA).

Cell cycle assay. A total of $1 \times 10^{6}$ cells were harvested and resuspended in $1 \mathrm{ml}$ McCoy's 5A (Modified) Medium supplemented with $10 \%$ FBS. Cells were stained with $2 \mu \mathrm{l}$ Vybrant DyeCycle Green Stain and incubated at $37^{\circ} \mathrm{C}$ for $30 \mathrm{~min}$. The cell cycle was analyzed using flow cytometry using $488 \mathrm{~nm}$ excitation and green emission.

Cell viability assays. Following treatment with IR, cells were mixed with $10 \%$ volume of alamarBlue reagent (Invitrogen; Thermo Fisher Scientific, Inc.) and incubated for $30 \mathrm{~min}$ at $37^{\circ} \mathrm{C}$, protected from direct light. Results were recorded using florescence at 570/585 nm (excitation/emission).

Cell apoptosis assay. Cells were harvested and washed twice with cold phosphate-buffered saline. Subsequently, cells were resuspended in 1X binding buffer (BD Biosciences, San Jose, CA, USA) at a concentration of $1 \times 10^{6}$ cells $/ \mathrm{ml}$ and stained with PE Annexin V with 7-AAD (BD Biosciences) in the dark for $15 \mathrm{~min}$ at room temperature. Samples were analyzed using flow cytometry.

Statistical analysis. Statistical analysis was performed using Microsoft Excel 2010 (Microsoft Corp., Redmond, WA, USA).
Data are presented as the mean \pm standard error of the mean of three independent experiments. Differences in mean values between groups were determined by Student's t-test. $\mathrm{P}<0.05$ was considered to indicate a statistically significant difference.

$\gamma H 2 A X$ foci formation. A-204 cells were seeded onto glass slides. Following transfection with siRNAs, the cells were irradiated with 4 Gy for $30 \mathrm{~min}$, washed twice in PBS for $5 \mathrm{~min}$ and subsequently fixed for $10 \mathrm{~min}$ at room temperature using $4 \%$ paraformaldehyde. Cells were washed twice in PBS for $5 \mathrm{~min}$, permeated for $10 \mathrm{~min}$ at room temperature using permeabilisation buffer ( $0.2 \%$ TritonX-100 in PBS) and washed twice more for $5 \mathrm{~min}$. Cells were subsequently blocked for $1 \mathrm{~h}$ at room temperature with blocking buffer (0.05\% FBS in PBS), and incubated with anti- $\gamma \mathrm{H} 2 \mathrm{AX}$ (1:200; Merck KGaA, Darmstadt, Germany; 05-636-AF488) antibody overnight at $4^{\circ} \mathrm{C}$. The cells were washed twice more in washing buffer $(0.05 \%$ Tween in PBS) and the coverslips were mounted with mounting medium (glycerol), stained with DAPI and detected using a fluorescent microscope (Leica Microsystems GmbH, Wetzlar, Germany). $\gamma \mathrm{H} 2 \mathrm{AX}$ foci were analyzed using Image Pro Plus 6.0 software (Media Cybernetics, Inc., Rockville, MD, USA).

\section{Results}

Radiation induces CEP164 protein expression. To determine whether CEP164 is involved in the DDR, cells were $\gamma$-irradiated with 2, 4, 6 Gy and, following this, CEP164 expression levels were measured. Results demonstrated that CEP164 protein expression levels increased following IR with 2 and $4 \mathrm{~Gy}$ and the expression levels peaked at $24 \mathrm{~h}$ following treatment with 2 Gy IR in A-204 and A-673 cells, respectively (Fig. 1).

CEP164 inhibition induces G2/M arrest of the cell cycle. In order to determine whether CEP164 has a regulatory role in cell cycle progression, siRNA was utilized to knockdown CEP164 expression levels in A-204 and A-673 cells and, following $24 \mathrm{~h}, \mathrm{G} 2 / \mathrm{M}$ cell cycle arrest was measured. Results demonstrated that CEP164 delayed S-phase exit. Furthermore, to determine whether inhibition of CEP164 enhances the radiosensitivity of A-204 and A-673 cells, the cell cycle of CEP164-depleted cells following treatment with IR was analyzed. Treatment of A-204 cells with 2 Gy of IR induced entry into G2/M phase and a lower population in the synthesis (S) phase; however, combined radiation and CEP164 inhibition resulted in G2/M cell cycle arrest. These experiments were also conducted on A-673 cells, and similar results were found in CEP164 silencing A-673 cells (Fig. 2).

CEP164 silencing increases cell apoptosis and decreases cell viability upon IR. Following the result that CEP164 inhibition is able to arrest cells in the $\mathrm{S}$ phase of the cell cycle, colony formation assays were conducted in order to determine whether CEP164 has a role in cell proliferation and apoptosis. Cell viability assays indicated that CEP164 knockdown significantly decreased cell viability following treatment with IR compared with the negative control IR-treatment group $(\mathrm{P}<0.05)$. In order to determine whether CEP164 knockdown enhances IR-induced apoptosis, four different experimental conditions were investigated, including: No IR and no CEP164 
A
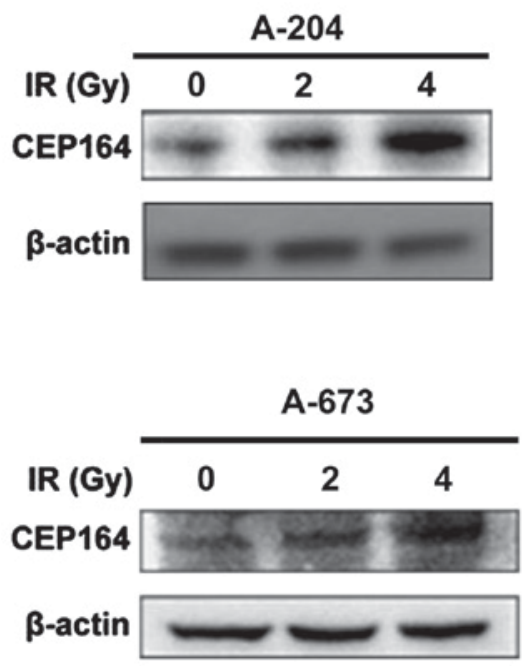

B
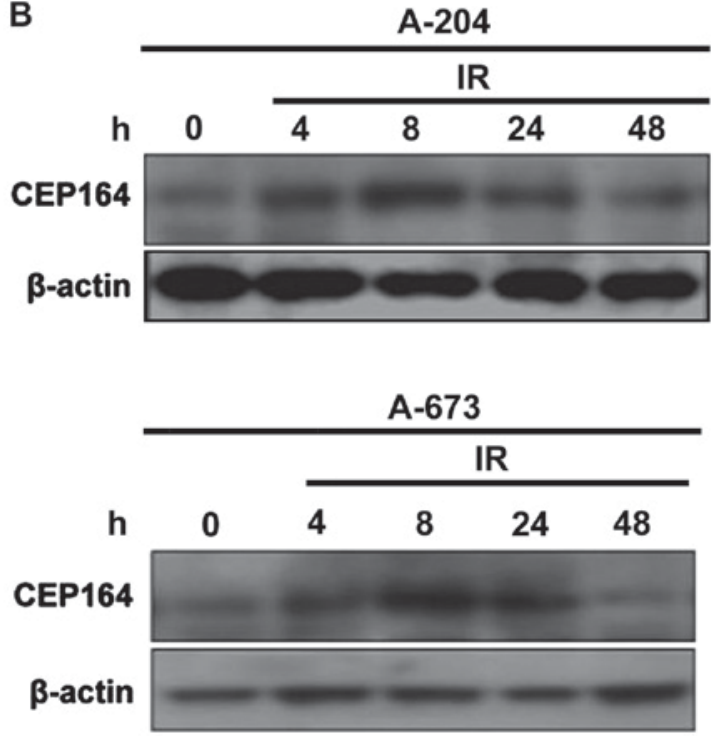

Figure 1. CEP164 protein expression levels increase following IR. (A) Western blot analysis of A-204 and A-673 rhabdomyosarcoma cells treated with IR of 2 and 4 Gy. (B) A-204 and A-673 rhabdomyosarcoma cells were exposed to 2 Gy IR and subsequently incubated for 4, 8, 24 and 48 h. CEP164, centrosomal protein 164; IR, irradiation.

A

A

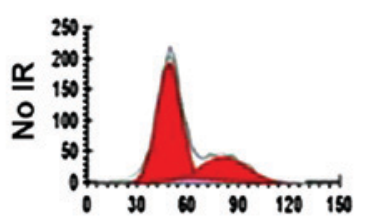

A-204

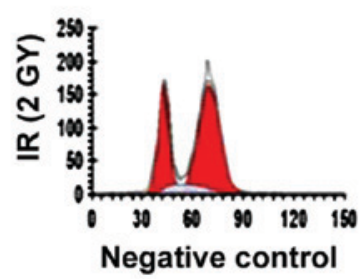

B

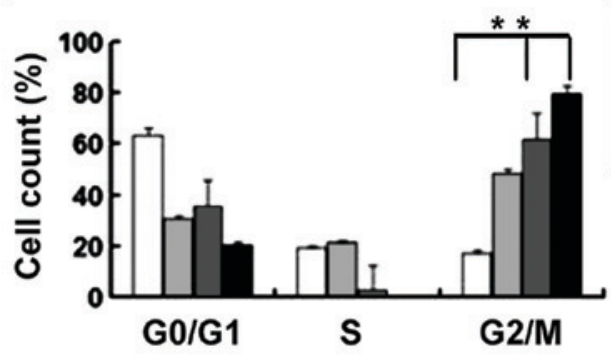

C

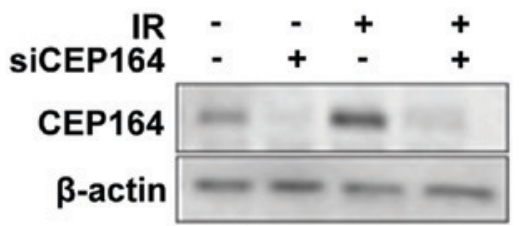

A-673
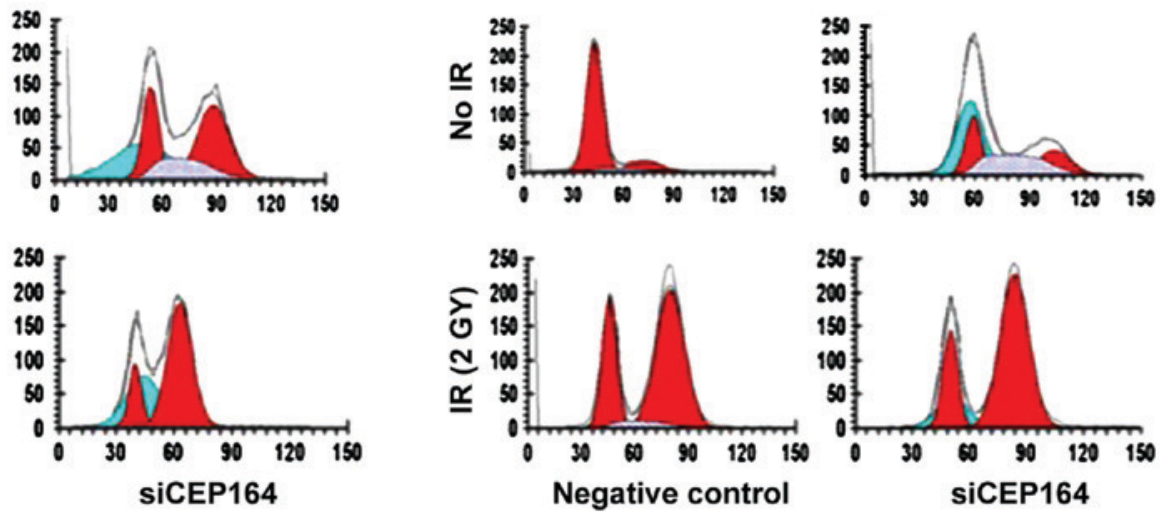

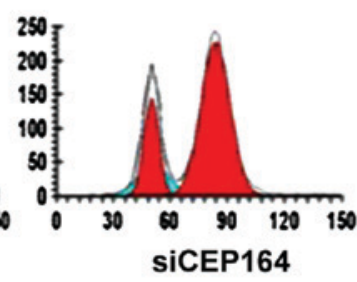

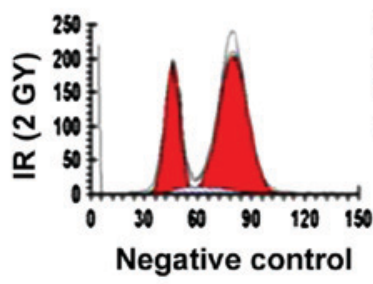
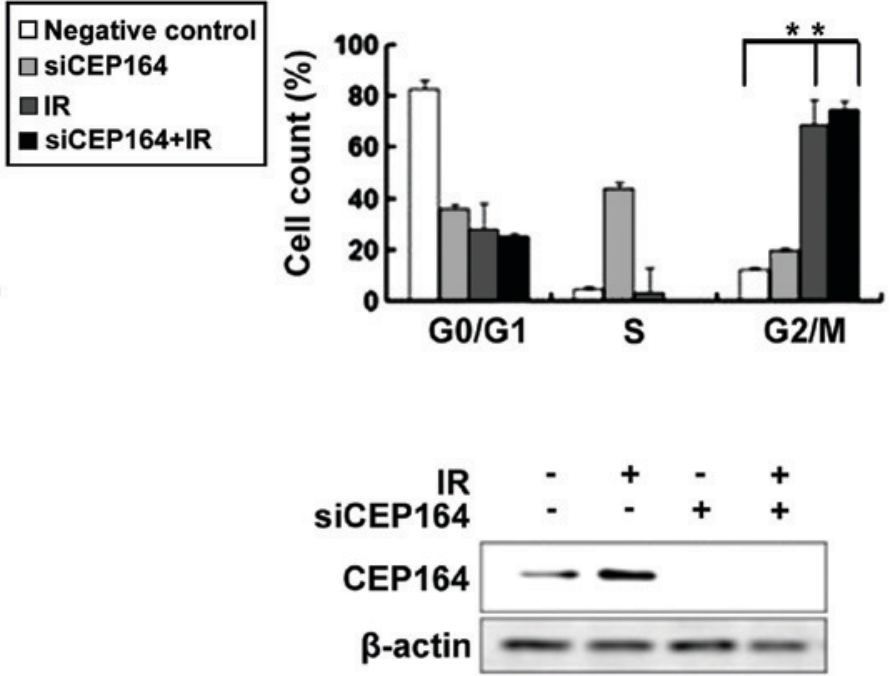

Figure 2. CEP164 deletion results in G2/M cell cycle arrest. (A) A-204 and A-673 rhabdomyosarcoma cells were transfected with siCEP164 RNA and subsequently treated with IR. Cell cycle progression was determined by DyeCycle staining. (B) Quantification of cell cycle assays. Similar results were obtained in three experiments. ${ }^{* *} \mathrm{P}<0.01$ vs. the negative control group. (C) Western blot analysis of CEP164 expression in CEP164-knockdown cells with or without IR (2 Gy). Data are presented as the mean + standard error of the mean. CEP164, centrosomal protein 164; IR, irradiation; si, small interfering; G0/G1, resting/gap 1; S, synthesis; G2/M, gap 2/mitosis. 
A

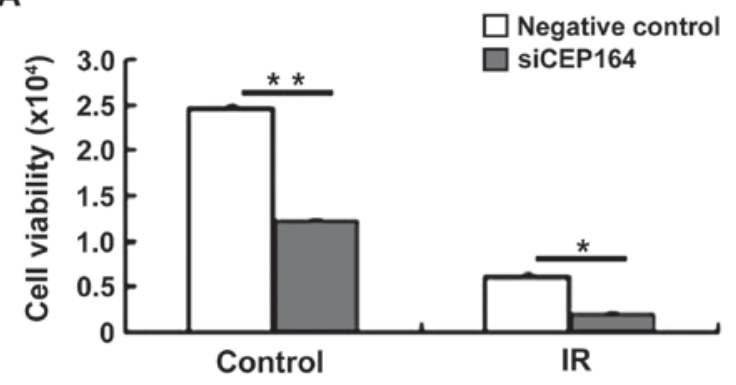

B

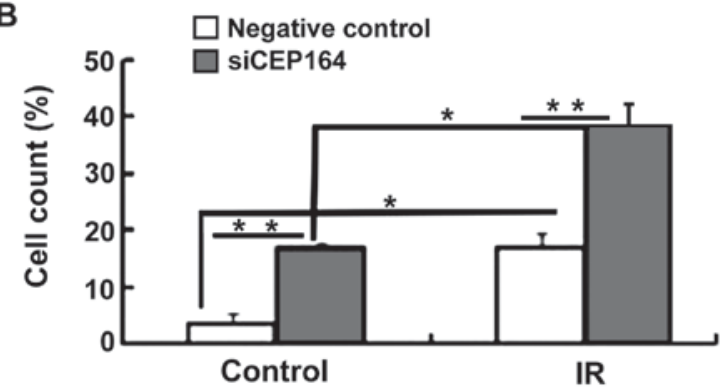

Figure 3. CEP164 silencing decreases cell viability and promoted apoptosis upon IR. (A) Relative cell viabilities of A-204 rhabdomyosarcoma cells were measured using alamarBlue staining. (B) siCEP164 transfected A-204 cells were exposed to $4 \mathrm{~Gy}$ of IR and cell apoptosis was subsequently quantified. Similar results were obtained in the three experiments. Data are presented as the mean + standard error of the mean. ${ }^{*} \mathrm{P}<0.05,{ }^{* * *} \mathrm{P}<0.01$ vs. the negative control group. CEP164, centrosomal protein 164; IR, irradiation; si, small interfering.

knockdown; IR only (4 Gy); CEP164 knockdown only; and combined CEP164 knockdown and IR (4 Gy). Results demonstrated that the combined treatment of CEP164-silencing and IR produced a significant increase in apoptosis compared with either of the treatments alone $(\mathrm{P}<0.05, \mathrm{P}<0.01$; Fig. 3). These results indicate that CEP164 is able to facilitate cellular proliferation and inhibit radiation injury.

CEP164 knockdown upregulates IR-induced $\gamma H 2 A$ histone family member $X(\gamma H 2 A X)$ foci formation. In order to confirm whether CEP164 is able to enhance the DDR, the $\gamma \mathrm{H} 2 \mathrm{AX}$ foci assembly in A-204 cells with or without CEP164 silencing was investigated. It was demonstrated that CEP164 depletion significantly increased $\gamma \mathrm{H} 2 \mathrm{AX}$ foci assembly compared with the negative control cells during 4 Gy IR $(P<0.05)$, indicating that CEP164 repression may enhance the recruitment of $\gamma \mathrm{H} 2 \mathrm{AX}$ and its associated repair proteins to DNA damage sites (Fig. 4).

\section{Discussion}

Cardiac tumors are classified as primary benign and malignant tumors if they arise in the heart, or as secondary tumors if they metastasize to the heart. In children, $90 \%$ of cardiac tumors are benign, whereas in adults $75 \%$ of cardiac tumors are benign (1,11-13). Rhabdomyosarcoma is the most common soft tissue malignant tumor in infants and children (14). Surgical resection of rhabdomyosarcoma is technically challenging and many malignant tumors cannot be resected completely. Chemotherapy and radiation therapy are able to relieve symptoms and prolong survival. A multi-treatment approach, including chemotherapy, radiation and novel,
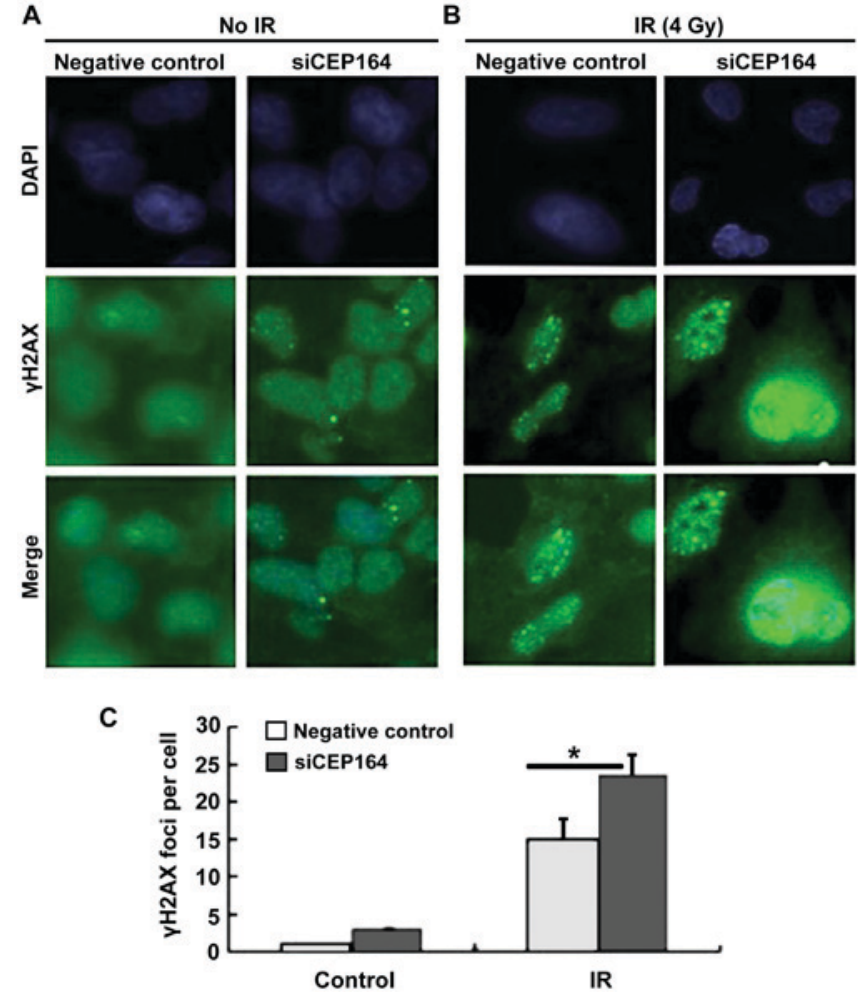

Figure 4. $\gamma \mathrm{H} 2 \mathrm{AX}$ foci formation. A-204 cells were stained with anti-phospho histone H2AX (Ser139) alexa fluor 488 conjugate antibody and observed using a fluorescent microscope (magnification, $\mathrm{x} 400$ ). (A) Left panel presents A-204 rhabdomyosarcoma negative control cells without IR treatment and the right panel indicates siCEP164 A-204 rhabdomyosarcoma transfected cells without IR treatment. (B) Left panel presents A-204 rhabdomyosarcoma negative control cells without CEP164 knockdown that were exposed to 4 Gy IR and the right panel indicates A-204 rhabdomyosarcoma cells with CEP164 knockdown that were exposed to 4 Gy IR. (C) $\gamma \mathrm{H} 2 \mathrm{AX}$ foci formation. The number of foci per nucleus were quantified. Data are presented as the mean + standard error of the mean for three independent experiments, ${ }^{*} \mathrm{P}<0.05$ vs. the negative control group. $\gamma \mathrm{H} 2 \mathrm{AX}, \gamma \mathrm{H} 2 \mathrm{~A}$ histone family member X; IR, irradiation; siCEP164, small interfering centrosomal protein 164

evolving approaches, such as gene therapy, may provide a more effective palliative and curative result.

CEP164 protein expression levels and activity are up-regulated in various types of cancer, and the protein is often overexpressed in tumors and is associated with poor prognosis (10). Additionally, CEP164 has a key role in primary cilia formation, which is a marker for distal appendages on mature centrioles or basal bodies $(3,6)$. As a novel mediator protein in DDR, CEP164 is required for the DNA damage-activated signaling cascade $(4,10)$. CEP164 interacts with both ATR and ATM, which are able to phosphorylate CEP164 under conditions of DNA damage and replication stress. Following phosphorylation, CEP164 is able to phosphorylate H2AX and CHEK2 (15). In the present study, the association of CEP164 knockdown with radiosensitivity in the cells was investigated. Results demonstrated that CEP164 protein expression levels increased following IR and inhibition of CEP164 resulted in the increased radiosensitivity of rhabdomyosarcoma cells. Furthermore, the results demonstrated that CEP164 promotes cell survival and decreases cell viability upon IR, indicating that CEP164 has an important role in cellular proliferation in response to cellular stress. 
CEP164 is required for genomic stability and CEP164 expression is cell cycle stage-dependent, being expressed at the end of the $\mathrm{S}$ phase and the beginning of the G2/M phase (16). CEP164 is essential for G2/M checkpoint activation through the phosphorylation of CHEK proteins. The results of the present study indicated that CEP164 engaged in cellular G2/M arrest, demonstrating that CEP164 has a function in cell cycle switching.

In conclusion, the present study demonstrated that CEP164 deletion is involved in the IR-induced cellular response and enhances the radiosensitivity of rhabdomyosarcoma cells. This, therefore, indicates that CEP164 may be a potential target to improve the outcome of radiotherapy in heart rhabdomyosarcoma treatment.

\section{References}

1. Yilmaz M, Kehlibar T, Arslan IY, Yilmaz HY, Tarhan IA and Ozler A: A case of primary cardiac rhabdomyosarcoma with surgical removal and mitral valve repair. Heart Surg Forum 16: E164-E166, 2013.

2. Abraham R: Cell cycle checkpoint signaling through the ATM and ATR kinases. Genes Dev 15: 2177-2196, 2001.

3. Čajánek L and Nigg EA: Cep164 triggers ciliogenesis by recruiting Tau tubulin kinase 2 to the mother centriole. Proc Nat Acad Sci USA 111: E2841-E2850, 2014.

4. Chaki M, Airik R, Ghosh AK, Giles RH, Chen R, Slaats GG, Wang H, Hurd TW, Zhou W, Cluckey A, et al: Exome capture reveals ZNF423 and CEP164 mutations, linking renal ciliopathies to DNA damage response signaling. Cell 150: 533-548, 2012 .
5. Chen K: Rhabdomyosarcoma in an adult presenting with nodal metastasis: A pitfall in fine-needle aspiration cytology of lymph nodes. Diagn Cytopathol 32: 303-306, 2005.

6. Graser S, Stierhof YD, Lavoie SB, Gassner OS, Lamla S, Le Clech M and Nigg EA: Cep164, a novel centriole appendage protein required for primary cilium formation. J Cell Biol 179: 321-330, 2007.

7. Guo L, Wang ZY,Zou YB and Bi LR: Primary cardiac embryonal rhabdomyosarcoma: Report of a case. Zhonghua Bing Li Xue Za Zhi 42: 621-622, 2013 (In Chinese).

8. Ma J, Sun JP, Chen M, Zhang L, Xu N, Wang J, Pui-wai Lee A and Yu CM: Left atrial rhabdomyosarcoma. Circulation 129: e503-505, 2014.

9. Merlino G and Helman LJ: Rhabdomyosarcoma-working out the pathways. Oncogene 18: 5340-5348, 1999.

10. Pan YR and Lee EY: UV-dependent interaction between Cep164 and XPA mediates localization of Cep164 at sites of DNA damage and UV sensitivity. Cell Cycle 8: 655-664, 2009.

11. Paulino AC and Okcu MF: Rhabdomyosarcoma. Curr Probl Cancer 32: 7-34, 2008

12. Sivasubramaniam S, Sun X, Pan YR, Wang S and Lee EY: Cep164 is a mediator protein required for the maintenance of genomic stability through modulation of MDC1, RPA, and CHK1. Genes Dev 22: 587-600, 2008.

13. Yang S, Gao Y, Zhao H and Cao D: Cardiac embryonal rhabdomyosarcoma in an adult. Eur J Cardiothorac Surg 45: e233, 2014.

14. Raphael S, Yusuf I and Imam I: Childhood rhabdomyosarcoma in Kano, Nigeria: A retrospective analysis of 52 cases. Niger J Med 24: 32-36, 2015.

15. Nigg EA, Čajánek L and Arquint C: The centrosome duplication cycle in health and disease. FEBS Lett 588, 2366-2372, 2014.

16. Slaats GG, Ghosh AK, Falke LL, Le Corre S, Shaltiel IA, van de Hoek G, Klasson TD, Stokman MF, Logister I, Verhaar MC, et al: Nephronophthisis-associated CEP164 regulates cell cycle progression, apoptosis and epithelial-to-mesenchymal transition. PLoS Genet 10: e1004594, 2014. 